\title{
SOLVING WEIGHTED NORM INEQUALITIES USING THE RUBIO DE FRANCIA ALGORITHM

\author{
STEVE BLOOM
}

\begin{abstract}
Given an operator $T$ bounded on a weighted $L^{p}$ space, the factorization technique of Rubio de Francia forces strong conditions on the weight. This algorithm is extended to two weight problems, and is shown to yield not just necessary but sufficient conditions in a wide range of settings.
\end{abstract}

A nonnegative weight $w \in A_{p}$ for some $p>1$ if for all intervals (or cubes) $I$,

$$
\frac{1}{|I|} \int_{I} w(x) d x\left(\frac{1}{|I|} \int_{I} w^{-1 /(p-1)}\right)^{p-1} \leq C,
$$

where $C$ is a universal constant independent of $I$. We will use the notation $w(I)$ for $\int_{I} w(x) d x$ and $I(w)$ for $w(I) /|I|$. Muckenhoupt studied these weights in detail, establishing, among other facts, that the Hardy-Littlewood maximal operator $f \rightarrow$ $f^{*}$ is bounded on $L^{p}(w d x)$ if and only if $w \in A_{p}$ [5]. A weight $w \in A_{1}$ provided

$$
I(w) \leq C \underset{I}{\operatorname{essinf}} w \text { for all intervals } I,
$$

or equivalently, $w^{*}(x)=\sup _{x \in I} I(w) \leq C w(x)$ a.e. Peter Jones [4] showed that $w \in A_{p}$ if and only if there exist weights $u$ and $v \in A_{1}$ with $w=u v^{1-p}$.

Recently, Jose L. Rubio de Francia applied Maurey's theory of factorization of operators to such weighted norm inequalities and obtained a very elegant proof of the Jones' factorization theorem [7]. This technique, which I have dubbed the Rubio de Francia Algorithm, or the RdFA, yields factorization theorems for many types of operators. Guido Weiss [10] observed that in the unweighted case, when the operator is an integral operator with nonnegative kernel, the condition forced by the RdFA corresponds to the hypotheses in Schur's lemma. So, the RdFA produces necessary and sufficient conditions for the boundedness of an operator.

In $\S 1$, we will apply the RdFA to the two-weight problem for such integral operators, obtaining necessary and sufficient conditions. In $\S 2$, we will look at a number of applications.

1. The RdFA. Let $X$ denote an appropriate measure space, $R^{n}$ for $n \geq 1$ or the unit circle in the complex plane. Let $\mu$ and $\lambda$ be nonnegative weight functions. $L^{p}(\mu)$ will denote $L^{p}(X, \mu(x) d x)$.

THEOREM (THE RDFA). Let $Q(x, y) \geq 0$ and let $T$ be the operator $T f(x)=$ $\int Q(x, y) f(y) d y$. Let $p>1$, with $1 / p+1 / q=1$. Then $T: L^{p}(\mu) \rightarrow L^{p}(\lambda)$ is a

Received by the editors June 30, 1986.

1980 Mathematics Subject Classification (1985 Revision). Primary 42B25; Secondary 42B20, $42 \mathrm{~A} 50$. 
bounded operator if and only if there exists a nonnegative function $\alpha \in L^{p q}$, with

(1) $\lambda^{1 / p} T\left(\alpha^{q} \mu^{-1 / p}\right) \leq C \alpha^{q}$ a.e. and

(2) $\mu^{-1 / p} T^{*}\left(\alpha^{p} \lambda^{1 / p}\right) \leq C \alpha^{p}$ a.e., where $T^{*}$ is the adjoint of $T$.

PrOOF. Let $S$ be the operator

$$
S f=\left(\lambda^{1 / p} T\left(f^{q} \mu^{-1 / p}\right)\right)^{1 / q}+\left(\mu^{-1 / p} T^{*}\left(f^{p} \lambda^{1 / p}\right)\right)^{1 / p} .
$$

Then $S$ is a bounded operator on $L^{p q}$, and $S$ is sublinear, by Minkowski's inequality. Let $K>\|S\|$. For any $f \in L^{p q}$ with $f \geq 0$, let $\alpha=\sum_{n=0}^{\infty} K^{-n} S^{n} f$, where $S^{n} f=S\left(S^{n-1} f\right), \alpha \in L^{p q}$, and by the sublinearity of $S$,

$$
S \alpha \leq K(\alpha-f) \leq K \alpha \text {. }
$$

Since the two operators comprising $S$ are nonnegative when applied to $\alpha$, we have (1) and (2).

Conversely, set

$$
M_{z} f=\lambda^{1 / p} \alpha^{-q+(p+q) z} T\left(\alpha^{q-(p+q) z} \mu^{-1 / p} f\right) .
$$

Since $\left|M_{x+i y} f\right| \leq M_{x}(|f|)$, this is an admissible family of operators on the strip $S=\{z: 0 \leq \operatorname{Re} z \leq 1\}$. If $f \in L^{\infty}$,

$$
\left|M_{0} f\right| \leq \lambda^{1 / p} \alpha^{-q}\|f\|_{\infty}\left(\alpha^{q} \mu^{-1 / p}\right) \leq C\|f\|_{\infty}
$$

by (1), while if $f \in L^{1}$,

$$
\begin{aligned}
\int\left|M_{1} f(x)\right| d x & \leq \int \lambda^{1 / p}(x) \alpha^{p}(x) \int Q(x, y) \alpha^{-p}(y) \mu^{-1 / p}(y)|f(y)| d y d x \\
& =\int \alpha^{-p} \mu^{-1 / p}|f(y)| \int Q(x, y) \lambda^{1 / p}(x) \alpha^{p}(x) d x d y \\
& =\int \alpha^{-p} \mu^{-1 / p}|f(y)| T^{*}\left(\lambda^{1 / p} \alpha^{p}\right)(y) d y \\
& \leq K\|f\|_{1}
\end{aligned}
$$

by (2). Now, complex interpolation $\left[9\right.$, p. 205] gives $M_{1 / p}$ bounded on $L^{p}$, that is

$$
\int\left|T\left(\mu^{-1 / p} f\right)\right|^{p} \lambda \leq C \int|f|^{p}
$$

or with $g=\mu^{-1 / p} f$,

$$
\int|T(g)|^{p} \lambda \leq C \int|g|^{p} \mu .
$$

2. Applications. We will apply the RdFA to four operators. The first two are integral operators with nonnegative kernels, so the RdFA applies directly. The other operators are not, so the theorem does not apply. Even so, an easy modification to the RdFA yields necessary conditions for $L^{p}$ boundedness of the operators, and again these conditions turn out to be sufficient.

The fractional integral. There has been a flurry of recent effort trying to characterize weight pairs for which the various fractional integrals

$$
I_{t} f(x)=\int|x-y|^{t} f(y) d y
$$


are $L^{p}$ bounded. This is an ideal setting for the RdFA. $I_{t}$ is a selfadjoint operator with nonnegative kernel, so $I_{t}: L^{p}(\mu) \rightarrow L^{p}(\lambda)$ is a bounded operator if and only if there exists an $\alpha \in L^{p q}$ with

$$
I_{t}\left(\alpha^{q} \mu^{-1 / p}\right) \leq C \alpha^{q} \lambda^{-1 / p} \quad \text { and } \quad I_{t}\left(\alpha^{p} \lambda^{1 / p}\right) \leq C \alpha^{p} \mu^{1 / p} .
$$

Hardy's inequality. Let $T f(x)=\frac{1}{x} \int_{0}^{x} f(y) d y$ on the domain $[0, \infty)$. This is an integral operator with the kernel $Q(x, y)=\frac{1}{x} \chi_{[0, x]}(y)$. That is of course nonnegative, so the RdFA applies. Its adjoint is the operator

$$
T^{*} f(x)=\int_{x}^{\infty} \frac{f(y) d y}{y} .
$$

According to the RdFA, $T: L^{p}(\mu) \rightarrow L^{p}(\lambda)$ is a bounded operator if and only if there exists an $\alpha \in L^{p q}$ with

$$
T\left(\alpha^{q} \mu^{-1 / p}\right) \leq C \alpha^{q} \lambda^{-1 / p}
$$

and

$$
T^{*}\left(\alpha^{p} \lambda^{1 / p}\right) \leq C \alpha^{p} \mu^{1 / p} .
$$

Muckenhoupt [6] showed that $T: L^{p}(\mu) \rightarrow L^{p}(\lambda)$ is bounded if and only if

$$
\sup _{x>0}\left(\int_{x}^{\infty} \lambda(y) y^{-p} d y\right)^{1 / p}\left(\int_{0}^{x} \mu^{-q / p}(y) d y\right)^{1 / q}<\infty .
$$

(1) and (2) are an interesting characterization of (3).

Suppose that $\mu=\lambda$. Then we have a Jones' type factorization: Let $u=\alpha^{p} \lambda^{1 / p}$ and $v=\alpha^{q} \lambda^{-1 / p}$. Then $u v^{-p / q}=\lambda$, where

$$
T^{*} u \leq C u \text { and } T v \leq C v .
$$

This is a necessary and sufficient condition for the boundedness of $T$ on $L^{p}(\lambda)$. The prototypical such $v$ looks like $v(x)=x^{\alpha-1}$ for some $\alpha>0$, while the typical $u$ looks like $u(x)=x^{-\beta}$ for some $\beta>0$. So such a good weight $\lambda$ looks like

$$
\lambda(x)=x^{-\beta}\left(x^{\alpha-1}\right)^{-p / q}=x^{p / q-t}
$$

for some $t>0$, and we have

$$
\int_{0}^{\infty}\left[\int_{0}^{x} f(y) d y\right]^{p} x^{-1-t} d t \leq C(t) \int_{0}^{\infty} x^{p}|f(x)|^{p} x^{-1-t} d x
$$

which is the classical Hardy's inequality.

There is no reason to restrict this one dimension. In $R^{n}$, let

$$
T f\left(x_{1}, x_{2}, \ldots, x_{n}\right)=\int_{0}^{x_{1}} \int_{0}^{x_{2}} \cdots \int_{0}^{x_{n}} f\left(y_{1}, y_{2}, \ldots, y_{n}\right) d y_{n} \cdots d y_{1} .
$$

The existence of an $\alpha$ for which (1) and (2) hold is necessary and sufficient for the boundedness of $T: L^{p}\left(R_{+}^{n}, \mu\right) \rightarrow L^{p}\left(R_{+}^{n}, \lambda\right)$.

As far as we know, this is the first characterization of a good weight pair for Hardy's inequality in more than two dimensions.

The Hardy-Littlewood maximal operator. One possible approach to the HardyLittlewood maximal operator is to linearize it, with $R(f)(x)=I_{x}(|f|)$, where $I_{x}$ is 
an interval containing $x$. This operator is an integral operator with kernel $Q(x, y)=$ $\chi_{2}(y) /\left|I_{x}\right|$, so the RdFA applies, but not well. The adjoint of this operator is not pleasant, and even worse, the function $\alpha$ produced by the RdFA depends on the linearization.

Still, it was in this setting that Rubio de Francia first applied his technique. Suppose $f \rightarrow f^{*}$ is bounded on $L^{p}(w d x)$ and on $L^{q}\left(w^{-q / p} d x\right)$. Let $S$ be the operator

$$
S f(x)=\left[w^{1 / p}\left(f^{q} w^{-1 / p}\right)^{*}(x)\right]^{1 / q}+\left[w^{-1 / p}\left(f^{p} w^{1 / p}\right)^{*}(x)\right]^{1 / p} .
$$

Then this is bounded on $L^{p q}$. For $f \in L^{p q}$, with $f \geq 0$, again define

$$
\alpha=\sum_{n=0}^{\infty} K^{-n} S^{n} f
$$

$\alpha$ is in $L^{p q}$ for $K>\|S\|$, and by the sublinearity of $S, S \alpha \leq K \alpha$. So $u=\alpha^{p} w^{1 / p}$, $v=\alpha^{q} w^{-1 / p} \in A_{1}$, and $w=u v^{1-p}$.

It is easy to see that such weights are in $A_{p}$, so Muckenhoupt's theorem shows that this condition is sufficient also.

What is particularly nice is that the interpolation argument in the RdFA can be modified to apply to this setting, to yield a proof that $f \rightarrow f^{*}$ is bounded on $L^{p}(w)$ and $L^{q}\left(w^{-q / p}\right)$ if and only if $w=u v^{1-p}$ for $u$ and $v \in A_{1}$.

The interpolation argument is as follows: Let $u$ and $v \in A_{1}$ and $w=u v^{1-p}$. Muckenhoupt's theorem is quite easy for $A_{1}$ weights, since

$$
I(|f|)=\frac{1}{|I|} \int_{I}|f| u^{-1} u \leq \frac{C}{u(I)} \int_{I}|f| u .
$$

Let $M_{u}^{*} f(x)=\sup _{x \in I}(1 / u(I)) \int_{I}|f| u$. The traditional Hardy-Littlewood theorem gives $M_{u}^{*}$ bounded on $L^{p}(u)$. Since $f^{*} \leq C M_{u}^{*} f$, we have $f \rightarrow f^{*}$ bounded on $L^{p}(u)$.

As C. Fefferman and Stein [3] observed, it suffices to establish the boundedness of the operator $f \rightarrow f_{d}^{*}$, the dyadic maximal operator. Let $M f(x)=\sup \{|I(f)|: x \in$ $I, I$ a dyadic interval $\}$. Since $f_{d}^{*}=M(|f|)$, it will suffice to bound $M$. Fix $f$ and $k \in Z$. Arguing much like Sawyer [8], let $\left\{I_{n}^{k}\right\}$ be the maximal dyadic intervals relative to the set $\left[M f>2^{k}\right]$. These are disjoint, $\left|I_{n}^{k}(f)\right|>2^{k}$, and $\left[M f>2^{k}\right]=\bigcup_{n} I_{n}^{k}$. Let $E_{n}^{k}=I_{n}^{k} \sim\left[M f>2^{k+1}\right]$. Then

$$
\begin{aligned}
\int(M f)^{p} w & =\sum_{k} \int_{2^{k}<M f \leq 2^{k+1}}(M f)^{p} w \\
& \leq 2^{p} \sum_{k} 2^{p k} \sum_{n} w\left(E_{n}^{k}\right) \leq 2^{p} \sum_{k} \sum_{n}\left|I_{n}^{k}(f)\right|^{p} w\left(E_{n}^{k}\right) .
\end{aligned}
$$

Let $f=g v u^{-1 / p}$. So $\int|f|^{p} w=\int|g|^{p} v$. We will have to show

$$
\sum_{k, n}\left|I_{n}^{k}\left(g v u^{-1 / p}\right)\right|^{p} w\left(E_{n}^{k}\right) \leq C \int|g|^{p} v .
$$

Now since $u \in A_{1}$

$$
I\left(|g| v u^{-1 / p}\right) \leq \frac{C}{I\left(u^{1 / p}\right)} I(v)\left[\frac{1}{v(I)} \int_{I}|g| v\right] .
$$


Let

and

$$
\gamma(k, n)=\frac{I_{n}^{k}(v)^{p}}{I_{n}^{k}\left(u^{1 / p}\right)^{p}} w\left(E_{n}^{k}\right)
$$

Then

$$
T g(k, n)=\frac{1}{v\left(I_{n}^{k}\right)} \int_{I_{n}^{k}} g v
$$

so we must show

$$
\int|M f|^{p} w \leq C \sum_{k, n} T(|g|)^{p}(k, n) \gamma(k, n)
$$

$$
\sum_{k, n}|T g(k, n)|^{p} \gamma(k, n) \leq C \int|g|^{p} v
$$

where $C$ here does not depend on $\gamma$ (which depended on $f$ ). We want to bound $T: L^{p}(v) \rightarrow L^{p}(\gamma)$. Now $T: L^{\infty}(v) \rightarrow L^{\infty}(\gamma)$ with operator norm 1 . So all that needs be shown is that $T$ acts appropriately on $L^{1}$, using whatever interpolation theorem happens to strike your fancy. For instance, it is not hard to show that $T$ is of weak-type $(1,1)$. For this, if $\alpha$ is large and $g \geq 0$, by the CalderónZygmumd decomposition, there exist maximal disjoint dyadic intervals $\left\{I_{i}\right\}$ with $\alpha<\left(1 / v\left(I_{i}\right)\right) \int_{I_{i}} g v$ and with $g \leq \alpha$ a.e. off $\bigcup_{i} I_{i}$. Fix an $I=I_{i}$. If $I_{n}^{k} \cap I \neq \varnothing$, then either $I_{n}^{k} \subset I$ or $I \varsubsetneqq I_{n}^{k}$. In the latter case, and also when $I_{n}^{k}$ does not intersect any $I_{i}, T g(n, k) \leq \alpha$. Now

$$
\begin{aligned}
\frac{1}{v(I)} \sum_{I_{n}^{k} \subset I} \gamma(k, n) & =\frac{1}{v(I)} \sum_{I_{n}^{k} \subset I} \int_{E_{n}^{k}} I_{n}^{k}(v)^{p} I_{n}^{k}\left(u^{1 / p}\right)^{-p} w(x) d x \\
& \leq \frac{C}{v(I)} \sum_{I_{n}^{k} \subset I} \int_{E_{n}^{k}} I_{n}^{k}\left(u^{1 / p}\right)^{-p} \underset{I_{n}^{k}}{\operatorname{essinf}} v^{p} u(x) v^{1-p}(x) d x \\
& \leq \frac{C}{v(I)} \sum_{I_{n}^{k} \subset I} \int_{E_{n}^{k}} I_{n}^{k}\left(u^{-1 / p}\right)^{p}\left(\underset{I_{n}^{k}}{\operatorname{essinf} v}\right) u(x) d x
\end{aligned}
$$

by Cauchy-Schwarz,

$$
\begin{aligned}
& \leq \frac{C}{v(I)} \sum_{I_{n}^{k} \subset I} \int_{E_{n}^{k}} I_{n}^{k}\left(v^{1 / p} u^{-1 / p}\right)^{p} u(x) d x \\
& \leq \frac{C}{v(I)} \int_{I}\left(v^{1 / p} u^{-1 / p}\right)_{I}^{* p} u(x) d x
\end{aligned}
$$

where $f_{I}^{*}(x)=\sup \{J(|f|): x \in J \subset I\}$.

Here too, $C$ does not depend on $\gamma$, but only on the $A_{1}$ norm of $v$. As we pointed out earlier, Muckenhoupt's theorem holds for $A_{1}$ weights, so

$$
\frac{1}{v(I)} \sum_{I_{n}^{k} \subset I} \gamma(k, n) \leq \frac{C}{v(I)} \int_{I}\left(v^{1 / p} u^{-1 / p}\right)^{p} u=C,
$$

where this $C$ depends on $u$ as well as $v$, but not on $\gamma$. Hence,

$$
\gamma([(k, n): T g(k, n)>\alpha]) \leq C \sum_{i} v\left(I_{i}\right) \leq C \sum_{i} \frac{1}{\alpha} \int_{I_{i}} g v \leq \frac{C}{\alpha}\|g\|_{L^{\prime}(v)}
$$

and the proof is finished. 
This proof uses very little of the structure of $A_{p}$ weights. The only property needed showed up twice, in the hypotheses to both the Calderón-Zygmund decomposition and the Hardy-Littlewood theorem, the measure $d \mu$ must be a doubling measure, that is

$$
\mu([x-2 h, x+2 h]) \leq C \mu([x-h, x+h]) \text { for all } h>0 \text { and } x .
$$

This is immediate for measures $d \mu=u d x$, when $u \in A_{1}$. In particular, the proof does not use the reverse Hölder inequality of $A_{p}$ weights, the truly deep ingredient in Muckenhoupt's proof. This proof should extend, almost verbatim to settings in general spaces of homogeneous type, even when reverse Hölder fails.

The RdFA can be applied to Sawyer's two-weight problem also. If the operator $f \rightarrow f^{*}: L^{p}(\mu) \rightarrow L^{p}(\lambda)$, and $L^{q}\left(\lambda^{-q / p}\right) \rightarrow L^{q}\left(\mu^{-q / p}\right)$ boundedly, then there exists an $\alpha \in L^{p q}$ with

$$
\left(\alpha^{q} \mu^{-1 / p}\right)^{*} \leq C \alpha^{q} \lambda^{-1 / p} \quad \text { and } \quad\left(\alpha^{p} \lambda^{1 / p}\right)^{*} \leq C \alpha^{p} \mu^{1 / p} .
$$

It is not known whether these conditions are sufficient.

Commutators. For functions $b$, define the commutator operators

$$
S_{b} f(x)=\sup _{x \in I}|b(x) I(f)-I(b f)|
$$

and

$$
T_{b} f(x)=\left|b(x) \tilde{f}(x)-(b f)^{\sim}(x)\right|,
$$

where $f \rightarrow \tilde{f}$ is the conjugate operator. For $S_{b}$, intervals can be pushed up to cubes in $\mathbf{R}^{n}$, but for $T_{b}$, we will stick to one dimension. Let $\mu$ and $\lambda$ be nonnegative weights. The triple $(b, \mu, \lambda)$ is said to be an $L^{p}$ commutator triple for the maximal operator provided $S_{b}: L^{p}(\mu) \rightarrow L^{p}(\lambda)$ is bounded. Similarly, it is an $L^{p}$ commutator triple for the conjugate operator if $T_{b}$ is bounded. The problem of characterizing all such triples is quite delicate, and so far has only partially been resolved.

$T_{b}$ is an integral operator, but its kernel is not nonnegative. $S_{b}$ is even worse. There is no reason to hope that the RdFA will yield anything useful in this setting. Yet, in the cases resolved so far, the RdFA produces necessary and sufficient conditions!

When $\mu$ and $\lambda \in A_{p}$, these triples are fully understood. $(b, \mu, \lambda)$ is an $L^{p}$ commutator triple for either operator if and only if $b \in \mathrm{BMO}_{\left(\mu \lambda^{-1}\right)^{1 / p}}$, that is

$$
I(|b-I(b)|) \leq C I\left(\mu^{1 / p} \lambda^{-1 / p}\right)
$$

for all $I[\mathbf{1}, \mathbf{2}]$.

For $S_{b}$, the RdFA proceeds like this: Suppose $S_{b}: L^{p}(\mu) \rightarrow L^{p}(\lambda)$ is bounded. Since $\lambda \in A_{p}$, the operator

$$
M f=\lambda^{1 / p}\left(\lambda^{-1 / p} f\right)^{*}+\lambda^{1 / p} S_{b}\left(\mu^{-1 / p} f\right)
$$

is bounded on $L^{p}$, so there exists an $\alpha \in L^{p}$ with

$$
\lambda^{1 / p}\left(\lambda^{-1 / p} \alpha\right)^{*} \leq C \alpha \quad \text { and } \quad \lambda^{1 / p} S_{b}\left(\mu^{-1 / p} \alpha\right) \leq C \alpha .
$$

Let $u=\mu^{-1 / p} \alpha$ and $\nu=\left(\mu \lambda^{-1}\right)^{1 / p}$. We have $u \nu \in A_{1}$ and $S_{b} u \leq C u \nu . u \in L^{1}$ by Hölder's inequality, and

$$
\begin{aligned}
|b(x)-I(b)| I(u) & \leq|b I(u)-I(b u)|+|I(b u)-I(b) I(u)| \\
& \leq S_{b} u(x)+I\left(S_{b} u\right) \leq C(u \nu)(x) .
\end{aligned}
$$


So $S_{b}: L^{p}(\mu) \rightarrow L^{p}(\lambda)$ boundedly forces the existence of a $u \in L^{1}$ with $u \nu \in A_{1}$ and $|b(x)-I(b)| \leq C u(x) \nu(x) / I(u)$.

Conversely, any such $b$ satisfies

$$
I(|b-I(b)|) \leq C I(u \nu) I\left(u^{-1}\right) \leq C(\underset{I}{\operatorname{essinf}} u \nu) I\left(u^{-1}\right) \leq C I(\nu),
$$

or $b \in \mathrm{BMO}_{\nu}$. So the condition is sufficient.

For $T_{b}$, we look at the operator

$$
f \rightarrow \mu^{1 / p}\left(\mu^{-1 / p} f\right)^{*}+\lambda^{1 / p} T_{b}\left(\mu^{-1 / p} f\right) .
$$

This is bounded on $L^{p}$ and the RdFA yields an $\alpha \in L^{p}$ with $u=\mu^{-1 / p} \alpha \in A_{1}$ and $\left(T_{b} u\right) / u \nu \in L^{\infty}$.

Conversely, if $T_{b} u / u \nu \in L^{\infty}$ for some $u \in A_{1}$, Theorem 2.6 in [2] gives $b \in \mathrm{BMO}_{\nu}$, so this condition also is sufficient.

So, the RdFA easily produces necessary and sufficient conditions for weighted norm inequalities involving integral operators with nonnegative kernels. Quite often, even with more delicate operators, the algorithm produces necessary and sufficient conditions. The RdFA is a very handy investigative tool.

\section{REFERENCES}

1. S. Bloom, A commutator theorem and weighted BMO, Trans. Amer. Math. Soc. 292 (1985), 103-122.

2. __ Applications of commutator theory to weighted BMO and matrix analogs of $A_{2}$ (to appear).

3. C. Fefferman and E. M. Stein, Some maximal inequalities, Amer. J. Math. 93 (1971), 107-115.

4. P. Jones, Factorization of $A_{p}$ weights, Ann. of Math. (2) 111 (1980), 511-530.

5. B. Muckenhoupt, Hardy's inequality with weights, Studia Math. 44 (1972), 31-38.

6. Weighted norm inequalities for the Hardy maximal function, Trans. Amer. Math. Soc. 165 (1972), 207-226.

7. J. L. Rubio de Francia, Factorization and extrapolation of weights, Bull. Amer. Math. Soc. (N.S.) 7 (1982), 393-396.

8. E. Sawyer, A characterization of a two-weight norm inequality for maximal operators, Studia Math. 75 (1982), 1-11.

9. E. M. Stein and G. Weiss, Fourier analysis on Euclidean spaces, Princeton Univ. Press, Princeton, N.J., 1971.

10. G. Weiss, Various remarks concerning Rubio de Francia's proof of Peter Jones' factorization theorem and some applications of the ideas in this proof, preprint.

Department of Mathematics, Siena College, Loudonville, New York 12211 